\title{
6 INTERACCIÓN Y VISUALIZACIÓN DE DATOS EN EL PERIODISMO ESTRUCTURADO
}

\section{Interaction and data visualization in structured journalism}

\section{Pere Freixa, Mario Pérez-Montoro y Lluís Codina}

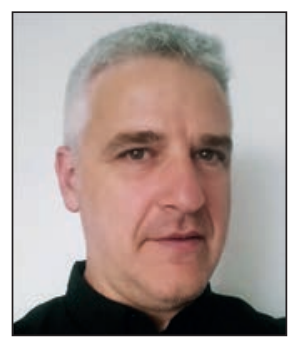

Pere Freixa es profesor del Departamento de Comunicación de la Universitat Pompeu Fabra. Es doctor en bellas artes por la Universitat de Barcelona y master en multimedia interactivo por la Universitat Politècnica de Catalunya. Forma parte de DigiDoc, grupo de investigación consolidado Agaur, como especialista en imagen fotográfica, interfaz gráfica e interacción. Dirige y ha participado en numerosos masters y posgrados especializados en investigación visual, interactividad y diseño interactivo. Dirige la revista académica Hipertext.net. http://orcid.org/0000-0002-9199-1270

Universidad Pompeu Fabra, Departamento de Comunicación Roc Boronat, 138. 08018 Barcelona, España pere.freixa@upf.edu

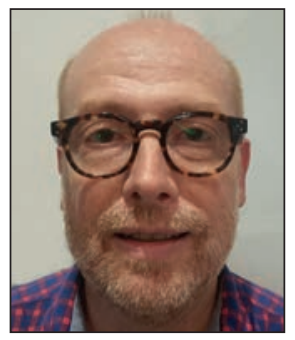

Mario Pérez-Montoro es doctor en filosofía y ciencias de la educación por la Universitat de Barcelona y posgraduado en organización de sistemas de documentación por la Universitat Politècnica de Catalunya. Ha realizado estudios de posgrado en el Instituto di Discipline della Comunicazione de la Universitá di Bologna (Italia), y ha sido profesor visitante del CSLI (Center for the Study of Language and Information) de la Stanford University (California, EUA) y de la School of Information de la UC Berkeley (California, EUA). Investiga sobre diseño de interacción y visualización de la información. Es profesor del Departamento de Biblioteconomía, Documentación y Comunicación Audiovisual de la Universitat de Barcelona.

http://orcid.org/0000-0003-2426-8119

Universitat de Barcelona, Departamento de Biblioteconomía, Documentación y Comunicación Audiovisual Melcior de Palau, 140. 08014 Barcelona, España perez-montoro@ub.edu

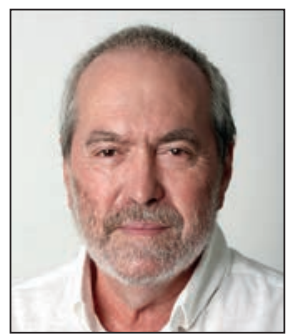

Lluís Codina es profesor de la Universitat Pompeu Fabra de Barcelona. Imparte docencia en la Facultad de Comunicación, en los grados de Periodismo y de Comunicación Audiovisual. Es coordinador del Master Universitario en Comunicación Social (MUCS) de esa universidad e imparte docencia también en los masters universitarios online en Documentación Digital y en Buscadores, del Instituto de Educación Continua. Es miembro promotor del grupo de investigación DigiDoc, y coordinador de su Seminario de investigación, así como del Observatorio de Cibermedios. Forma parte de la Unidad de Investigación en Periodismo, grupo consolidado reconocido por la Generalitat de Cataluña.

http://orcid.org/0000-0001-7020-1631

Universidad Pompeu Fabra, Departamento de Comunicación Roc Boronat, 138. 08018 Barcelona, España Iluis.codina@upf.edu

\section{Resumen}

La confluencia en las redacciones digitales de especialistas en narración interactiva, visualización de la información, documentación digital y periodismo de investigación ha permitido el auge del periodismo estructurado (structured journalism en inglés), una corriente muy reciente en el periodismo digital que contempla la reutilización, la acumulación de datos, la revisión y el contraste entre información actual e información archivada. Se define como un sistema en el que se combinan los recursos de almacenamiento y acceso a la información propios de las bases de datos con la potencia de explotación y difusión del periodismo digital. Se trata de una forma de aproximarse al contenido informacional en la que se prima la accesibilidad directa a la información primaria, los documentos y los datos, y en la que la construcción del relato narrativo queda 
mediatizada por la interactividad que cada usuario realiza cuando consulta la pieza informativa. Además de la obtención de información y la redacción de noticias, el periodismo estructurado precisa de una eficiente definición y estructuración de la base de datos en la que esa información se almacena, el diseño de capas e información, y de un relato interactivo.

\title{
Palabras clave
}

Periodismo estructurado; Periodismo; Periodismo digital; Medios digitales; Periodismo nativo digital; Géneros periodísticos; Interactividad; Datos; Visualización de información; Diseño de información.

\begin{abstract}
The confluence of professionals specializing in interactive storytelling, information visualization, digital documentation and investigative reporting in digital newsrooms has allowed the rise of the so called structured journalism (SJ). SJ is a recent trend in digital journalism that considers the reuse, accumulation, revision and contrast of current information with archived information and associated metadata. It is defined as a system in which the information storage in databases is combined with the potential for exploitation and diffusion of digital journalism. It is a way of approaching the informational content in which direct accessibility to primary information, documents, and data is given priority, and in which the construction of the narrative is mediated by the user's interactivity. In addition to obtaining information and writing news, structured journalism requires an efficient definition and structuring of the database in which that information is stored, the design of information layers and an interactive story.
\end{abstract}

\section{Keywords}

Structured journalism; Journalism; Online journalism; Online media; Native digital journalism; Journalism genres; Interactivity; Data; Information visualization; Information design.

Freixa, Pere; Pérez-Montoro, Mario; Codina, Lluís (2017). “Interacción y visualización de datos en el periodismo estructurado". El profesional de la información, v. 26, n. 6, pp. 1076-1090.

https://doi.org/10.3145/epi.2017.nov.07

\section{Introducción}

Desde mediados de la década de 2000 académicos, periodistas y centros de investigación están intentando definir y acuñar terminología que permita clasificar las formas emergentes de periodismo derivadas de la confluencia del ámbito digital y de la ciencia de la información, entre otras:

- periodismo de datos (data journalism o big data journalism) (Gray; Chambers; Bounegru, 2012; Lewis; Westlund, 2015);

- periodismo computacional, (computational journalism o, inicialmente, journalism via computer programming) (Holovarty, 2006; Hamilton; Turner, 2009; Daniel; Flew, 2010; Flew et al., 2012; Staverlin, 2014);

- periodismo estructurado (structured journalism) (Chua, 2010a);

- visualización de información (visual data) (Cleveland, 1993);

- remezcla de noticias (mashups) (Spoerri, 2007; Bradshaw, 2008);

- periodismo automático (automated journalism) (Caswell; Dörr, 2017);

- narrativa de datos interactiva (interactive data narrative) (Craig, 2015).

Todo ello refiere a productos periodísticos en los que los autores utilizan recursos relacionados con las bases de datos, algoritmos y programación para confeccionar el ítem informativo. Se consumen a través de dispositivos digitales en medios de comunicación o redes sociales, bien con el uso de navegadores o con aplicaciones (apps) específicas. En todo caso, precisan de una interfaz que permita la interacción y navegación de los usuarios.

\subsection{Objetivos y metodología}

En este artículo presentamos una aproximación al periodismo estructurado (PE), definiendo los rasgos que lo caracterizan. Para ello utilizamos una combinación de metodologías como la revisión bibliográfica y el análisis de caso múltiple. Como unidades de estudio se han seleccionado cinco ejemplos de PE. En una primera fase exploratoria, se ha realizado una revisión preliminar exhaustiva de obras que sus autores identifican con el PE, y finalmente, se ha seleccionado aqueIlas que consideramos que ofrecen mayor valor ilustrativo y posibilidad de estudio (Stake, 1995; Yin, 2013).

\subsection{Marco teórico}

Reg Chua, editor del proyecto Connected China y responsable de innovación de Reuters, expone las razones que justifican el periodismo estructurado:

"la esencia del periodismo estructurado reside en cambiar la forma en que creamos los contenidos con el fin de maximizar su vida útil, así como en estructurar, en el momento de su creación (...), la información en historias para que su uso en bases de datos sirva de fundamento para nuevas historias o productos de información" (Chua, 2010a).

http://china.fathom.info

Por su parte el BBC News Lab expone en un manifiesto publicado en 2015 las razones que les lleva a apostar por el periodismo estructurado:

“En primer lugar, explorar cómo facilitar a los periodistas, en su trabajo cotidiano, la captura de datos estructurados sobre los principales motores de las noticias; y en segundo lugar, construir cosas sobre datos estruc- 
turados que permitan a las audiencias explorar las noticias, entenderlas mejor y hacer el mismo uso de los datos que haría un periodista" (BBC News Lab, 2015).

El término periodismo estructurado (PE) aporta características que van más allá de un uso tecnológico o del deseo de destacar una parte del proceso que interviene en una determinada creación periodística. Para algunos autores la transformación principal radica en realizar cambios en los hábitos de trabajo y en los sistemas de gestión de los contenidos, que deben dejar de focalizarse en la noticia, entendida ésta como el ítem básico periodístico, para empezar a contemplar lo informativo como una estructuración de contenidos relacionados con las fuentes que los proveen y con los metadatos que se les puede asignar (Cohn, 2014).

Se trata de transformar el modo de elaboración de noticias diarias en una estructura que permita la reutilización posterior (Amico, 2013). La idea fundamental reside en crear procedimientos que permitan maximizar la vida de las noticias, convirtiendo la información en historias estructuradas en el momento de su creación, para convertirla en material de archivo fuertemente codificado, organizado en bases de datos, que permita convertir esa información en fundamento de futuras historias y productos periodísticos (Chua, 2010a).

Wojcik (2015) matiza que no debe confundirse periodismo estructurado con periodismo de datos. Según ella, el periodismo de datos parte de un volumen mayor o menor de información que se convierte en historia. Generalmente se trata de información no periodística proveniente de datos numéricos y estadísticos, que para convertirse en noticia se transforman en relato visual o textual. La visualización de datos resultante permite añadir valor social a la información, al transformar datos a menudo especializados en relatos comprensibles para la mayoría de lectores (Sánchez-Bonvehí; Ribera, 2014).

El periodismo estructurado parte de historias que se codifican para servir de base a nuevas historias, y el material original de que se parte es periodístico

En su lugar, el PE parte de historias que se codifican para servir de base a nuevas historias, y el material original de que se parte es periodístico. Es un procedimiento informativo en el que el sistema se retroalimenta periódicamente con nuevos datos. Debe resultar escalable (Chua, 2010b), permitir almacenar datos rutinarios que posteriormente y por acumulación, se transformen en noticia.

Para Bill Adair, director del Duke Reporters' Lab, solamente podemos hablar de PE cuando la información se actualiza constantemente (Owen, 2015). En su laboratorio están testando el software StructuredStories, un sistema de gestión de contenidos (CMS) diseñado por Yahoo para permitir la generación de relatos periodísticos a partir de los contenidos almacenados en su base de datos (Caswell; Russell; Adair, 2015). Los autores señalan que están desarrollando un proyecto conjunto con la ciudad de Nueva York con el propósito de gestionar la información municipal (Adair, 2015). http://www.structuredstories.com/\#/intro

Para Holovarty (2006) y Adair (2015), la característica principal que define el periodismo estructurado reside en el cambio de los sistemas de codificación de la información en las redacciones, los CMS, y en el aprendizaje de nuevas praxis por parte de los periodistas. Cambiar los procedimientos tradicionales basados en cuerpos de texto y titulares, por otros que permitan codificar con mayor precisión los datos propios de los acontecimientos, como el lugar, la fecha, los personajes, etc., permitirá mejorar la gestión, la búsqueda y la vinculación entre informaciones. Estas transformaciones presuponen un redactado de la información estandarizado, pautado, en el que se reportan las temáticas, los actores, las situaciones y los lugares involucrados en el acontecimiento del que se está informando.

Sin embargo, algunos proyectos insignia del PE basados de forma exclusiva en la codificación de información y generación automatizada de datos no han prosperado ni han tenido el impacto que se esperaba de ellos. La empresa Circa, abanderada de la atomización y estructuración del contenido (Cohn, 2014) entró en crisis en 2014, después de varios años sin conseguir superar los 1.000 usuarios.

https://www.circa.com

Tampoco prosperó la aplicación NYT Now de The New York Times que clausuró en 2016 (Lichterman, 2016). Para Newton (2015), Circa ejemplifica como el PE no siempre responde a las necesidades del periodismo de actualidad ya que el sistema puede provocar un exceso de neutralidad informacional y generalización, en lugar de proporcionar experiencias emocionales a los lectores.

Esta situación parece haberla resuelto favorablemente Jim Brady en Billy Penn, un medio digital pensado para público joven e información local. Promueve un periodismo estructurado basado en la colaboración y ensamblaje de contenidos, en el que el medio actúa como paraguas que da cobijo al conjunto de informaciones que se van relacionando con el evento principal (Stearns, 2015). Spirited Media, la productora de Billy Penn, está reproduciendo el modelo en otras ciudades, como Denver y Pittsburgh.

https://billypenn.com

https://spiritedmedia.com

En su modelo, la estructuración de la información permite la combinación de información generada por la redacción y la curaduría de datos provenientes de redes y activismo social que se ensamblan, en un intento por interconectar la información con los intereses de los colectivos jóvenes (Popper, 2015).

En todo caso, el PE está transformando algunos medios tradicionales. Laura Amico, coautora de Homicide watch DC, se ha incorporado a The Boston Globe para potenciar este modelo periodístico (Gourarie, 2015). En The Washington Post, Sarah Sampsel ha diseñado The knowledge map, una herramienta que permite añadir capas de información complementaria al hilo narrativo principal de la noticia.

http://homicidewatch.org 
En este sentido, Sampsel apunta:

"queríamos experimentar con el suministro de información complementaria y contextual en el momento en que un usuario lee una historia complicada; para ello diseñamos el mapa de conocimientos para trabajar de una manera que no interrumpa la experiencia de lectura" (WashPostPR, 2015).

Se trata de un complemento enciclopédico que permite explotar informaciones almacenadas en la base de datos del medio y que se retroalimenta con las nuevas informaciones que se codifican a diario.

Además de la sistematización de la información en bases de datos, el PE se caracteriza por incorporar la narración interactiva y la visualización de información, de modo que ambos son elementos que lo definen. Los dos aspectos juegan un papel fundamental, no solamente en el acabado del producto informativo, sino sobre todo en la definición y codificación de los datos, ya que con ello se determina el trazado y navegabilidad que el usuario podrá realizar, así como los parámetros que se podrán transformar en información visual. Brady considera que la tarea principal de la redacción de Billy Penn consiste en diseñar una buena experiencia de usuario, y que esta se basa en una buena narrativa interactiva (Popper, 2015).

En los grandes reportajes es donde el PE despliega todo su potencial. En Connected China, lanzado por Reuters en 2013, Ren Chua y el equipo de redacción diseñaron un proyecto en el que no se puede desasociar el diseño de interacción de las visualizaciones que se presentan en la interfaz ni de la estructura informativa. El producto informacional resultante aparece como un sistema complejo y dinámico, capaz de incorporar nuevos datos y de permitir al usuario la construcción de sus propios recorridos y relatos. Durante 18 meses recopilaron información que codificaron en la base de datos y que permitió crear el sistema. El diseño de las visualizaciones se realizó con criterios periodísticos, potenciando las soluciones que permitían hacer visibles interrelaciones complejas (Qiu; Zhang, 2013).

\section{Buenas prácticas y ejemplos}

Hay unanimidad en considerar el proyecto Chicagocrime. org de 2005, como predecesor e inspirador de lo que posteriormente hemos identificado como periodismo estructurado, aunque estrictamente se trate de un mashup, ya que se basó en vincular los mapas de Google Maps con datos sobre el crimen de la policía de Chicago sindicados por medio de RSS (Pisani, 2006; OHern; Rindfleisch, 2010; Flores-Vivar; Salinas-Aguilar, 2012). Obtuvo el premio Batten 2005 por su innovación periodística.

http://www.j-lab.org/projects/knight-batten-awards-forinnovations-in-journalism/knight-batten-2005

\section{El periodismo estructurado despliega} todo su potencial en los grandes reportajes

Su autor, Adrian Holovarty, destaca la labor pública que supuso el proyecto y su repercusión ciudadana (Holovarty, 2008). La relación de los datos sobre criminalidad publicados por la administración con mapas permitía a los lectores-usuarios interactuar con esas informaciones y generar significaciones propias según su relación con la ciudad (GiIlmor, 2006).

Como puede observarse en la figura 1, Chicagocrime.org se ofrece a los usuarios como un sitio web completo, autónomo, con dominio propio e independencia respecto a los medios de comunicación. En la interfaz se organiza la infor-
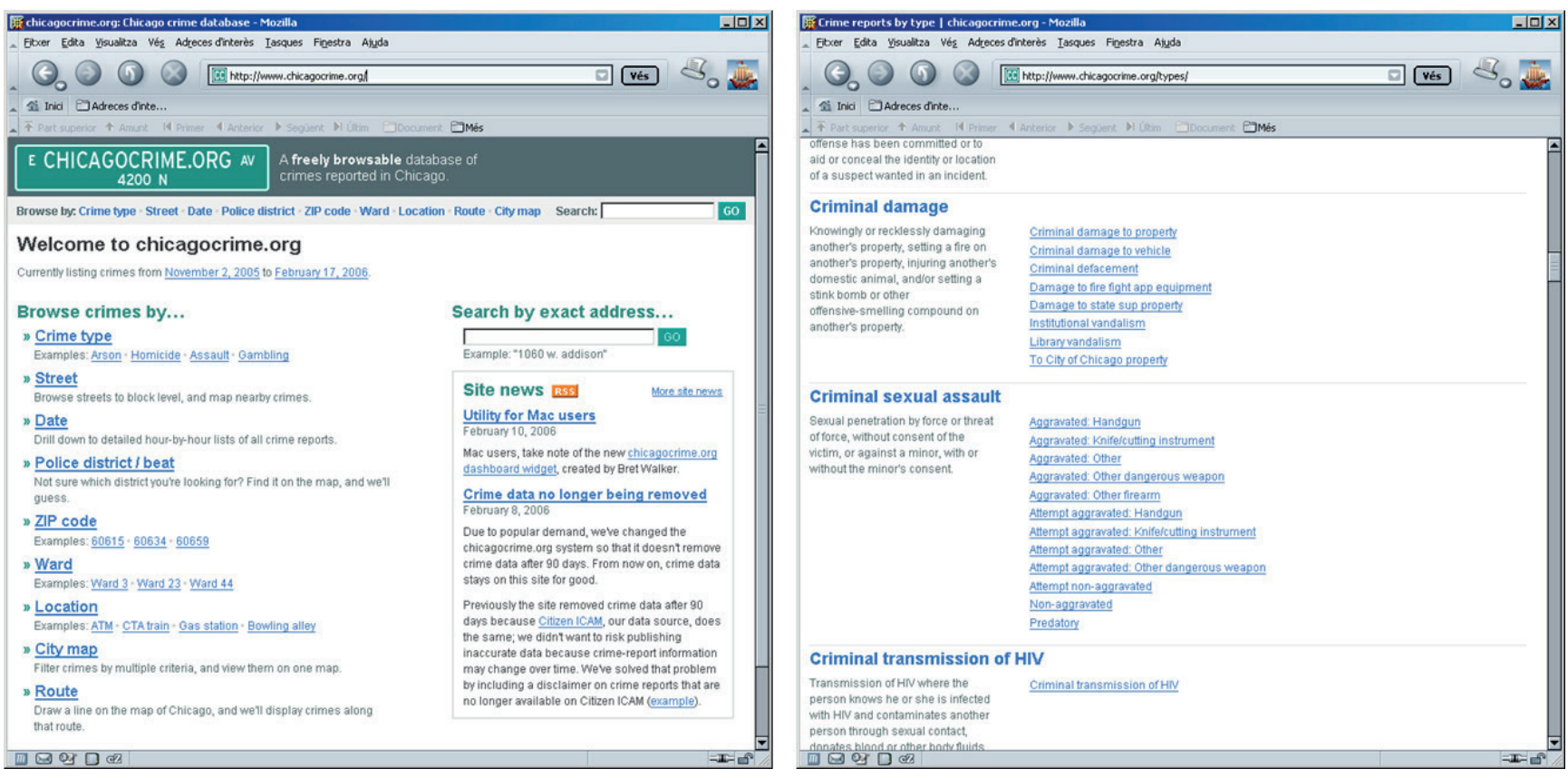

Figura 1. Captura de la interfaz principal y de la interfaz de "categorías" de la web Chicagocrime.org. Capturas realizadas en febrero de 2006 



Figura 2. Captura de la interfaz correspondiente a "Criminal defacement" y de la interfaz correspondiente al caso "HM184218" de la web Chicagocrime. org. Capturas realizadas en febrero de 2006

mación en capas morfológicas, por categorías y apartados relacionados con el etiquetado y la codificación de esa información en la base de datos. La unidad básica de información que alimenta el sistema es el informe o registro de cada crimen acontecido en la ciudad. Holovarty recupera los campos de las fichas policiales y los relaciona en capas informativas para facilitar el acceso cruzado a la información.

\section{El proyecto Chicagocrime.org de 2005 es predecesor e inspirador de lo que pos- teriormente hemos identificado como periodismo estructurado}

La representación de los datos en la interfaz de Google Maps que se ha incrustado en la página web (figura 2) ofrece una representación visual de la distribución geográfica del crimen en los barrios y calles de la ciudad, dibujando en su conjunto un complejo mapa sobre la criminalidad en Chicago. Hasta la fecha de cierre del proyecto en 2008, la aplicación se actualizaba con los nuevos datos que la policía publicaba periódicamente. La combinación de accesibilidad, estructuración de información y visualización de datos geolocalizados permite infinitos recorridos interactivos de los que emerge significación. El sistema da respuesta a las cuestiones que plantean los conciudadanos, como saber qué delitos se cometen cerca del colegio de los hijos o la peligrosidad de la zona en la que se vive. El sistema acaba revertiendo sobre la propia ciudad cuando los vecinos empiezan a utilizar Chicagocrime.org en sus quejas y reclamaciones vecinales (Holovarty, 2008).

Deudor de Chicagocrime.org, Homicide watch D.C., iniciado en 2010 por Laura Amico, se ofrece como mejora y se inscri- be plenamente en el periodismo estructurado. http://homicidewatch.org

Se clausuró en diciembre de 2014 debido a la falta de financiación (Amico, 2015), a pesar de cosechar numerosos premios y reconocimientos. El proyecto ha continuado en Chicago bajo el auspicio de Chicago Sun Times en colaboración con la Northwestern University's Medill School of Journalism, con el título de Homicide watch Chicago. http://homicides.suntimes.com

A diferencia de Chicagocrime.org, Homicide watch D.C. incorpora información periodística a los datos policiales:

"Utilizamos informes originales, documentos de fuentes primarias y redes sociales para construir uno de los recursos públicos más completos del país en materia de delitos violentos" (Amico; Amico, 2011).

La sede en internet se expande con perfiles en las redes sociales (Facebook y Twitter) y el sistema promueve la participación y colaboración ciudadana.

La web se complementa con la incorporación de un calendario en el que se agendan las fechas de juicio en que están implicados víctimas y sospechosos. Lo más significativo del proyecto reside en la incorporación de información periodística generada por los autores y colaboradores del proyecto. Chris y Laura Amico emplazan el proyecto en el ámbito informacional y lo conciben como proyecto periodístico. Las noticias o informaciones aportadas son tratadas como unidades que se codifican y catalogan en la base de datos y se almacena junto a los documentos y fuentes originales. El diseño de la interfaz y la interacción permite al lector recorrer las capas de información que conforman el proyecto: los redactados periodísticos, las fuentes documentales judiciales y los comentarios, aportaciones y correos de otros usuarios, también incorporados al sistema. En la columna derecha de 


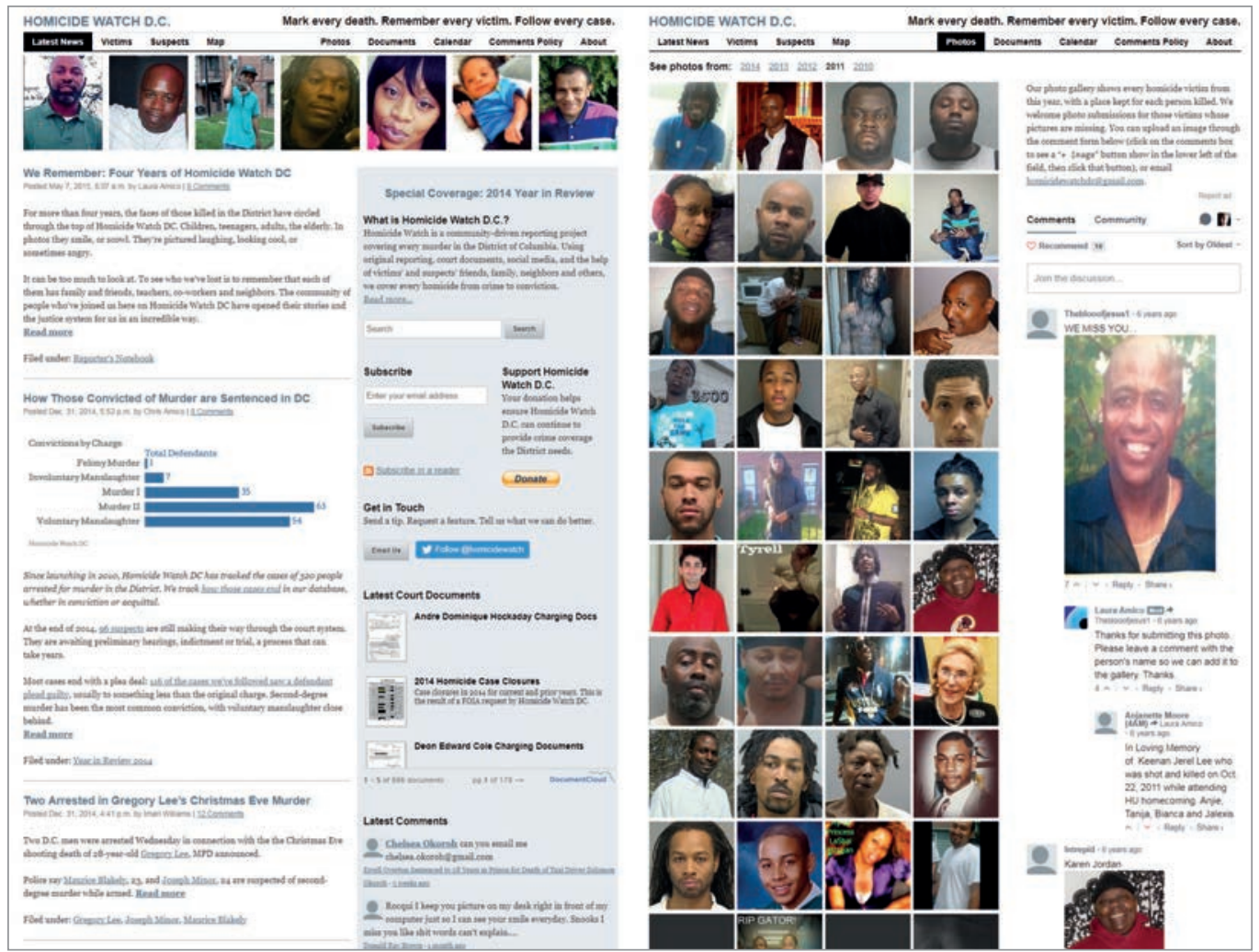

Figura 3. Captura de la interfaz principal y de la interfaz de "Photos" de la web Homicide watch D.C. Capturas realizadas en marzo de 2015

la figura 3 se puede apreciar el diálogo mantenido por la autora del proyecto con una usuaria relacionada con una de las víctimas.

El comentario periodístico se presenta junto al documento original escaneado del que se ha obtenido la información (figura 4). Cualquier nueva información relacionada con un determinado caso se vincula automáticamente con la ficha de ese homicidio, complementando las informaciones anteriores. EI PE supone contemplar el hecho informacional desde una perspectiva sistémica, en que las partes que conforman



Figura 4. Interfaz "Documents" e interfaz de "Victims" de la web Homicide watch D.C. Capturas realizadas en marzo de 2015 

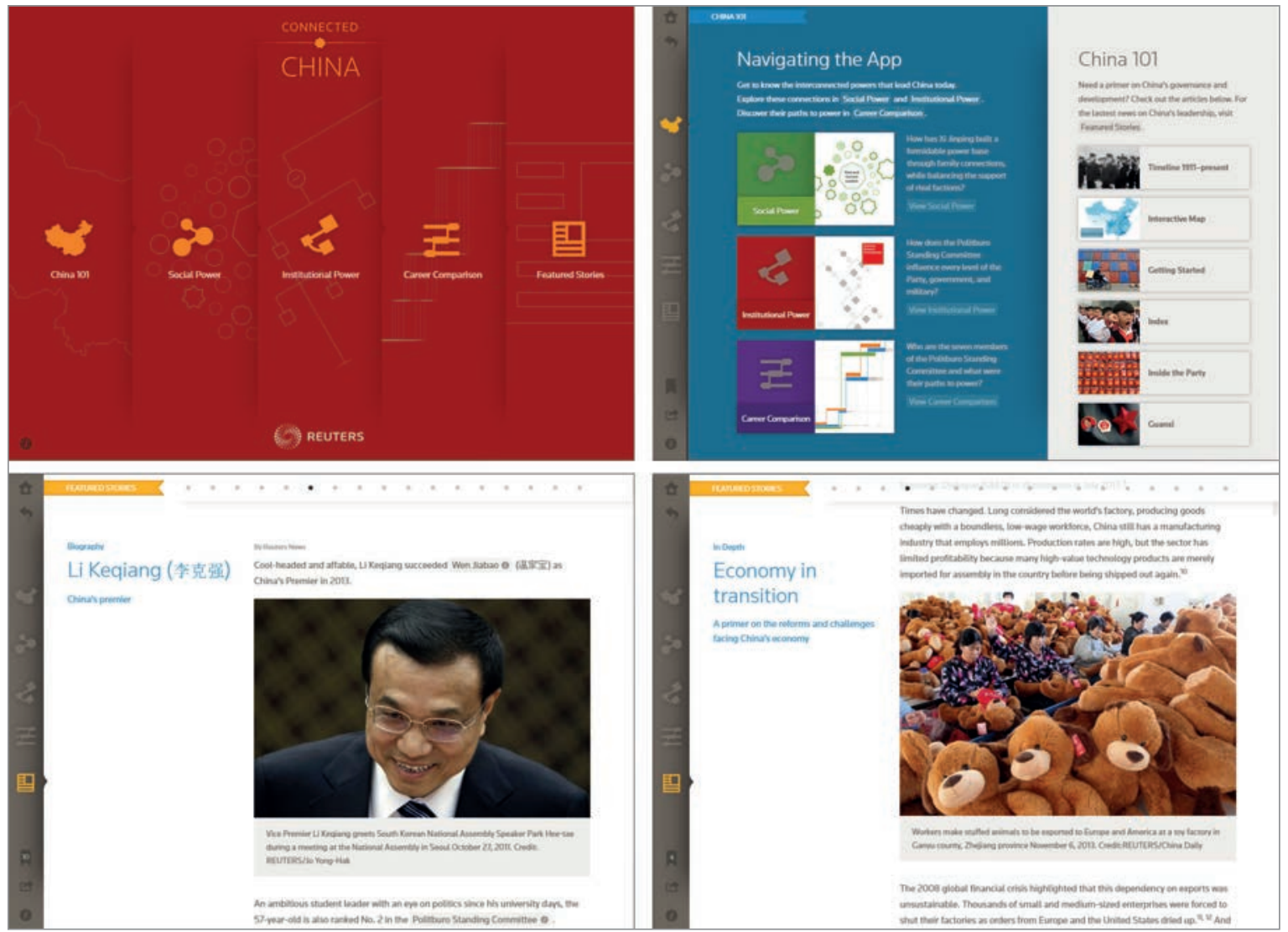

Figura 5. Conjunto de 4 capturas de pantalla de la interfaz del proyecto Connected China: "Pantalla principal”, "China 101" y dos ejemplos del apartado "Featured stories". Capturas realizadas en julio de 2017

el proyecto se retroalimentan y configuran una obra informacional exhaustiva. Supone concebir lo noticiable desde una dimensión temporal en que el relato periodístico sobrepasa la inmediatez del presente y se revaloriza como elemento de archivo que complementa el valor del conjunto.

Se trata de un sistema de periodismo de investigación que sobrepasa al reportaje tradicional. Cada vez que se accede a Homicide watch D.C. se reconoce la estructura, la temática y la misión del proyecto, aunque la información concreta sobre los casos y los personajes se haya actualizado y ampliado. El reaprovechamiento de la información y ciclo de vida informativo presente en otros formatos del periodismo digital (Freixa, 2015) se convierte en elemento definitorio del proceso informativo. Cada nuevo dato es susceptible de ser de utilidad y complementar datos posteriores. EI PE incorpora el archivo periodístico, la hemeroteca, como fundamento para construir las noticias que nos describen e informan sobre el presente.

El proyecto Connected China fue lanzado al mercado en febrero del 2013. Se trata de una aplicación informativa ideada para tabletas iOS (iPad2 o superiores) y navegadores web como Chrome y Safari.

http://china.fathom.info

Actualmente [julio 2017] se mantiene activo en la Red y es operativo en todos sus apartados, a pesar de que las últimas actualizaciones se realizaron en julio de 2014. Más allá del debate sobre el valor de la información mostrado en la aplicación, insuficiente para algunos (Kennedy, 2013) y excepcional para otros (Qiu; Zhang, 2013; Amico, 2013),
Connected China supone un punto de inflexión en el PE por varias razones. En primer lugar porque Reg Chua, responsable de innovación en Reuters y uno de los ideólogos del proyecto, pudo desplegar sin restricciones lo que él define por periodismo estructurado: la puesta en marcha de un sistema de codificación de información que permite la elaboración de productos informativos con mayor perdurabilidad que las informaciones convencionales gracias a la constante actualización de los datos que alimentan las aplicaciones que permiten a los usuarios su consulta. Según Irene Jay Liu, directora del proyecto:

"era importante que Connected China fuera interoperable con otros datos de Reuters (...) aunque la información en su base de datos subyacente cambiará, su principal promesa es que lo que muestra es preciso y actualizado, una imagen del liderazgo en China en ese preciso momento" (Amico, 2013).

El esfuerzo conjunto de Reuters y la empresa Fantom Design de Boston, por diseñar una aplicación atractiva para el espectador y adaptada a las prácticas del lenguaje audiovisual interactivo, permite alinear este producto informativo con la tradición de aplicaciones multimedia que vienen llevándose a cabo desde hace más de veinte años en otros ámbitos y que se fundamentan en el diseño de la interfaz y la interacción, la usabilidad y el diálogo con el usuario (Ribas; Freixa, 1997; Soler-Adillon et al., 2016). Con esta pieza se completa el PE desarrollado hasta entonces al otorgar valor comunicacional a la interacción que realiza el público. El proyecto destaca por el uso de un diseño visual adaptado a los datos, definiendo la interfaz y la visualización en función 

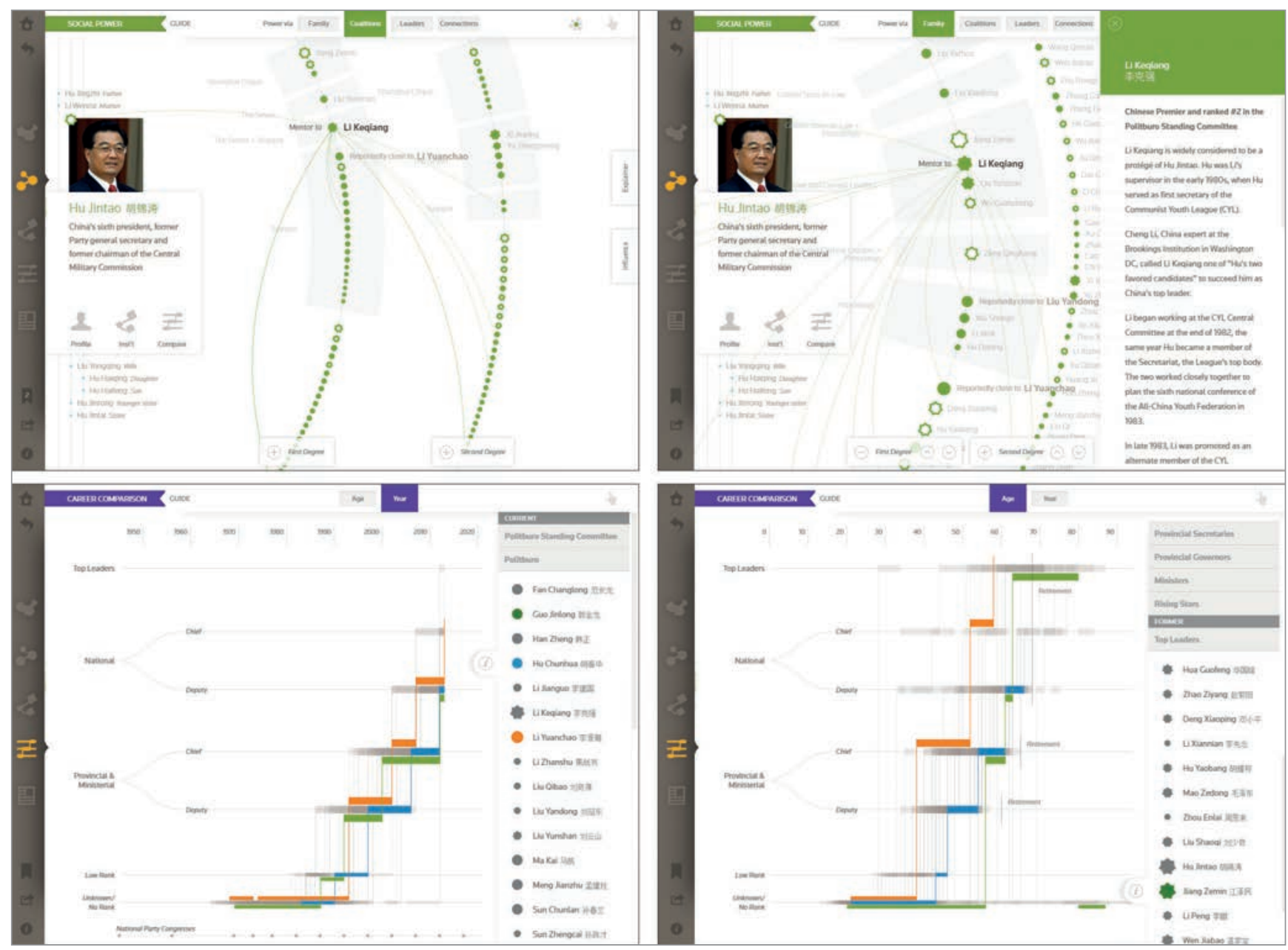

Figura 6. Conjunto de 4 capturas de pantalla del proyecto Connected China: dos del apartado "Social power" y dos de "Career comparison". Capturas realizadas en julio de 2017

del valor narrativo que se quiere conferir a la obra (figura 6). Se puede considerar que forma parte de la tradición de los especiales temáticos, construidos como sedes autónomas y con actualización de los datos, con más de diez años de presencia en los cibermedios (Larrondo, 2010).

\section{¿Cómo se consigue que un usuario regrese nuevamente a consultar una aplicación que se actualiza constantemente cuando su apariencia parece inmóvil?}

Como apunta Chris Amico, la dificultad para mantener el interés por Connected China reside en lo que a la vez le confiere mayor valor: aparentemente la aplicación siempre es la misma y resulta difícil adivinar qué ha variado en sus datos (Amico, 2013). ¿Cómo se consigue que un usuario regrese nuevamente a consultar una aplicación que se actualiza constantemente cuando su apariencia parece inmutable? Para ello resulta necesario que el medio y las redes sociales publiquen periódicamente nuevas informaciones que remitan al proyecto interactivo, revitalizando de este modo el interés por su consulta y exploración. Para ello, Reuters creó la cuenta@ConnectedChina en Twitter, aunque la dejó de actualizar al cabo de medio año.
Para finalizar este apartado de buenas prácticas y ejemplos, presentamos dos proyectos de ProPublica que permiten reflexionar sobre las características y los límites que definen el PE: ER wait watcher y Documenting hate.

ER wait watcher, publicado inicialmente en 2014 y que se mantiene actualizado actualmente en 2017 , se presenta al espectador en forma de sistema interactivo.

https://projects.propublica.org/emergency

Aparentemente se asemeja al mashup de Holovarty Chicagocrime.org: una interfaz funcional permite al usuario consultar datos estadísticos almacenados en una base de datos (figura 9, pantalla izquierda).

El usuario puede interactuar con la herramienta interactiva que ofrece datos sobre los tiempos de espera en los hospitales norteamericanos ordenados por Estados, por tiempo total de espera o por el tiempo que se tarda en recibir asistencia doméstica. La información se despliega con mayor detalle cuando se accede a los datos de un estado en concreto y, dentro de ese estado, a un centro médico específico (figura 9, derecha). Los usuarios puntúan y opinan sobre el servicio médico recibido. En la figura 9 se puede apreciar un comentario reciente, de mayo de 2017, en el que una usuaria maldice la espera que está soportando. 


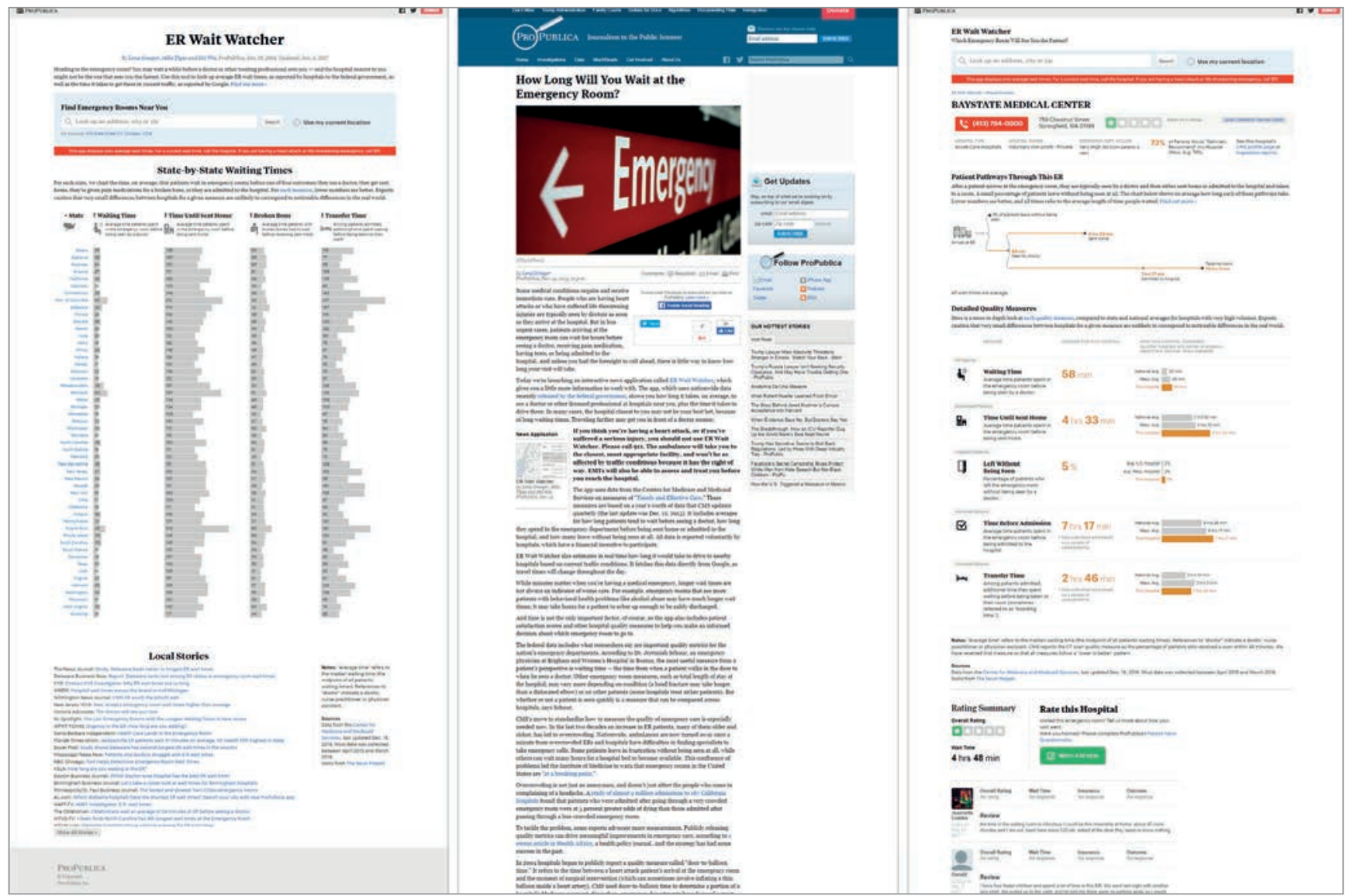

Figura 7. Capturas de 3 pantallas de ER wait watcher: pantalla principal, contenido ampliado y detalle de página dedicada a un centro médico. Capturas realizadas en julio de 2017

El mashup o visualización de datos se complementa con la información periodística que los redactores de ProPublica ofrecen en una segunda pantalla de contenido (figura 9, centro). La pieza finaliza con un apartado titulado "Local stories", en la parte inferior de la interfaz, en el que, como si de un programa de RSS se tratara, se enlazan artículos periodísticos propios y de otros medios relacionados con la temática del reportaje.

En esta pieza los límites entre visualización de datos, sindicación de información y periodismo estructurado pueden parecer difusos. A nuestro parecer, el interés de la pieza radica precisamente en esa combinatoria simultánea de elementos que juntos crean un producto informacional propio del PE.

Documenting hate fue iniciado en enero de 2017 por ProPublica y más de 80 medios, universidades y empresas de comunicaciones.

https://projects.propublica.org/graphics/hatecrimes

El trabajo se encuentra aún en fase de elaboración. Sus autores consideran que toma el relevo de Electionland, un macroproyecto ya finalizado que involucró a más de 250 medios, empresas y universidades (Grinapol, 2016). Monitorizó, recuperó datos y ofreció información en tiempo real de los problemas que se sucedieron en los comicios presidenciales de Estados Unidos en noviembre de 2016 (Hepworth, 2016).

https://projects.propublica.org/electionland
Los aprendizajes de coordinación, codificación de información, creación de interfaces, diseño del sistema informacional y dinámica de flujos de trabajo animó a ProPublica, Google News Labs, The New York Times, Meedan y el resto de socios a diseñar Documenting hate, un proyecto destinado a documentar y crear una gran base de datos en la que se reporte el crimen de odio, racista, de género y de prejuicios sociales en general (Trewinnard, 2017; The New York Times, 2017). En palabras de sus autores:

"Por primera vez seremos capaces de dar una mirada rigurosa a los crímenes de odio en Estados Unidos, combinando análisis de datos, recopilación de noticias de medios sociales y ambiciosas historias de investigación" (ProPublica, 2016).

Se trata de un proyecto de PE especialmente relevante porque pretende actuar en una doble vertiente. Por un lado, como recurso de información final, para su consulta y consumo por parte de los lectores; y por otro, como proveedor de información periodística para el conjunto de medios que participan en él. El proyecto se encuentra en una primera fase, de recogida y codificación de datos. En la figura 8 se puede apreciar la página principal. De momento presenta las entidades participantes, un primer índice de recopilación de noticias relativas al proyecto y la declaración de intenciones del mismo. En la figura 9 se pueden observar los formularios que se encuentran en los apartados "Get involved" y "Tell your story". Como puede comprobarse, la recopilación de información se realiza de forma estructurada. Con ello 

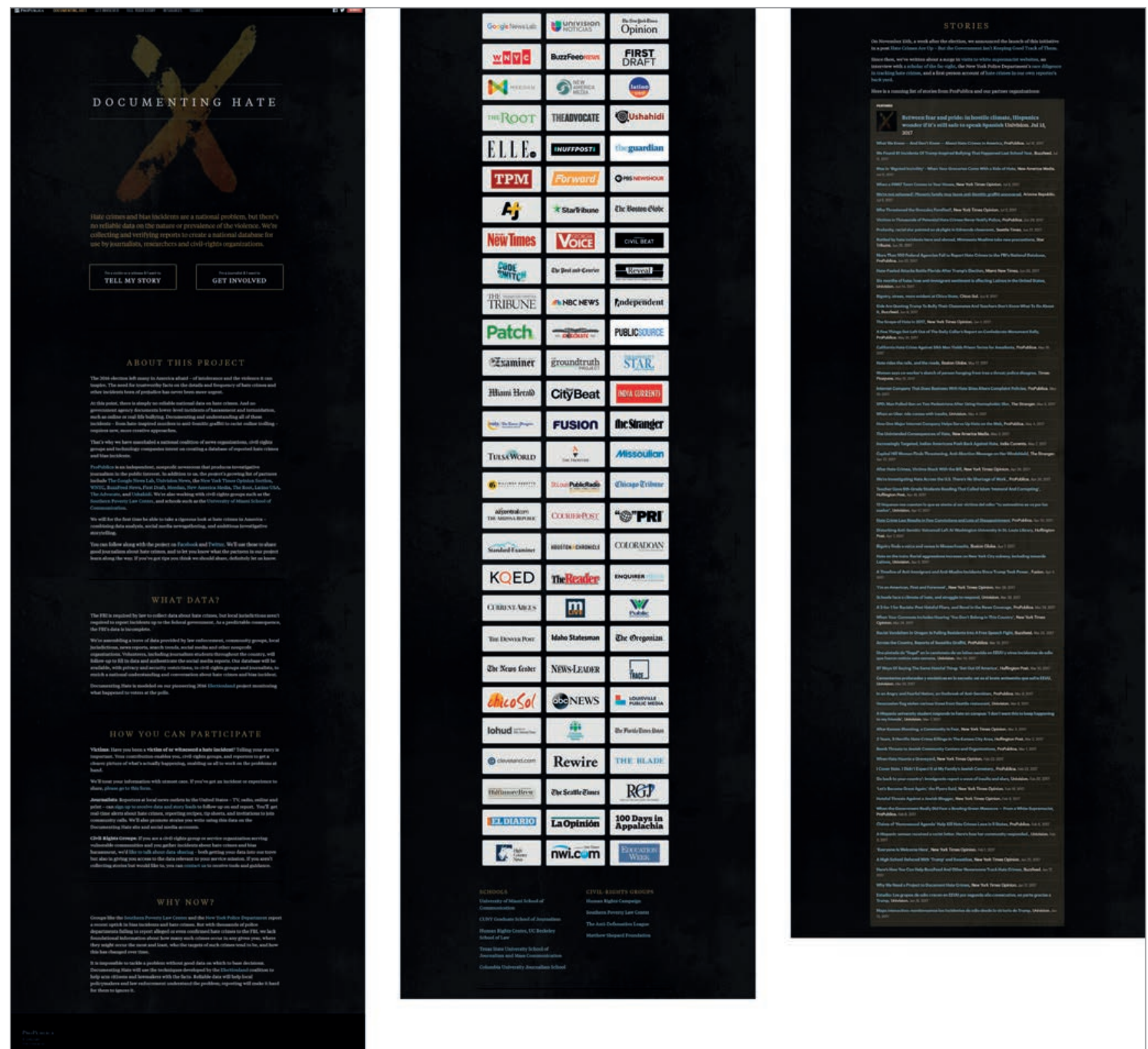

Figura 8. Captura de la pantalla principal del proyecto Documenting hate. Se ha dividido y editado en tres fragmentos -contenido, participantes y corpus de informaciones recopiladas- para facilitar su reproducción en esta página. Capturas realizadas en julio de 2017

se pretende poder validar la calidad de la información y codificar correctamente los datos. Univisión ha desarrollado una primera interfaz visual en la que presenta los datos del proyecto.

http://www.univision.com/noticias/incidentes-de-odio/ mapa-interactivo-monitoreamos-los-incidentes-de-odiodesde-la-victoria-de-trump

Se trata de un mapa interactivo en el que se van añadiendo paulatinamente las noticias reportadas. En la fase actual el proyecto resulta especialmente interesante ya que permite constatar cómo el PE resulta inoperante si no contempla los otros pilares que lo definen: el diseño de interacción, que posibilitará al usuario acceder y consultar las informaciones, y el diseño de interfaz y capas de información que permitirán estructurar y representar los datos y convertirlos en relato significante.

\section{Resultado: propuesta de caracterización}

¿Qué define entonces el periodismo estructurado? ¿Qué elementos lo conforman y caracterizan? a pesar de que bajo su nombre se da cobijo a productos, procedimientos y conceptos que pueden parecer dispares y que en gran medida aún se encuentran en estado de gestación, existen cinco factores que permiten su definición y le diferencian de otras formas de periodismo digital: sistema, datos, acceso, interactividad e interfaz (tabla 1 ).

\subsection{Información como sistema}

Tanto si se pretende dar cobertura de información diaria como de reportajes de gran formato, el PE precisa de una consideración sistémica por parte de sus autores y productores y de una previsión de ciclo de vida informativo, que contemple la explotación temporal del proyecto y de 

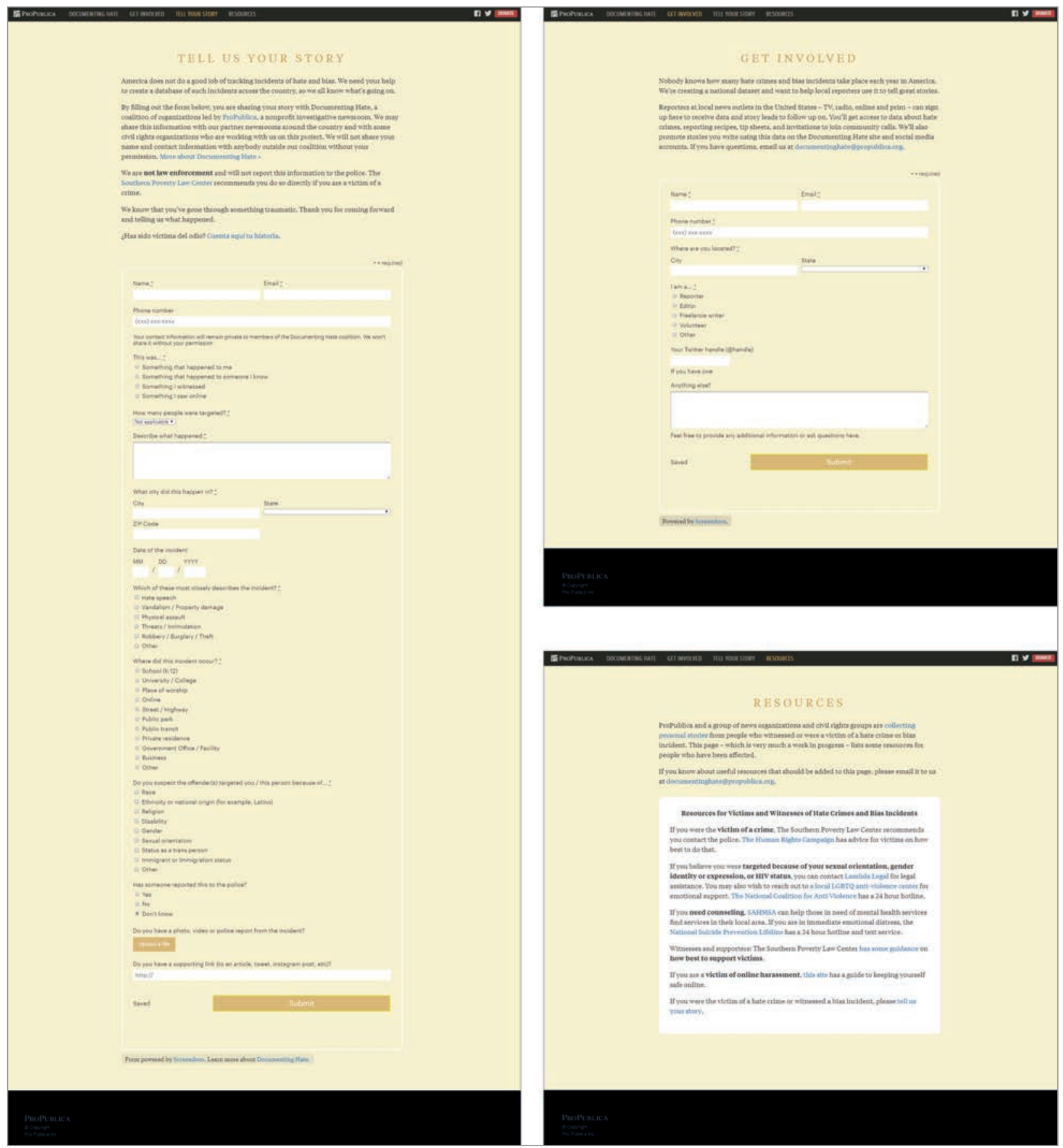

Figura 9. Formularios para participar en el proyecto Documenting hate en calidad de testimonio o colaborador. Capturas realizadas en julio de 2017

los contenidos informativos que contiene. El sistema debe prever su retroalimentación. La mayoría de proyectos de PE están diseñados para prolongarse indefinidamente, manteniendo en todo momento su capacidad de informar sobre el presente. Su vida útil depende en gran medida del interés de sus autores en mantener activo el sistema.

\subsection{Datos y codificación}

La mayoría de autores que defienden el periodismo estructurado esgrimen argumentos basados en la mejora que supone la codificación de la información y en la necesidad de transformar los programas tradicionales de gestión de contenido periodístico en potentes bases de datos que permitan la interrelación entre contenidos. En los últimos años, varios proyectos han intentado transformar completamente las redacciones periodísticas para construir sistemas basados en unidades de información que se recombinan para crear relatos periodísticos. En cualquier caso, se actúe a nivel global sobre todo un medio o a nivel de pieza informativa, el PE presupone codificar la información generada por los periodistas, los datos originales y los metadatos que se quieran añadir en una base de datos que permita su recuperación y tratamiento. Forman el nivel inferior de un sistema de PE. Una característica importante es que el sistema prevé la ampliación de la información de forma periódica, con aporte de nuevos datos.

\subsection{Acceso a la información por capas}

Si la información se almacena en unidades atomizadas codificadas con metadatos en bases de datos, para acceder a 
Tabla 1. Caracterización del periodismo estructurado

\begin{tabular}{|c|c|c|c|}
\hline & Elemento & Caracterización & Nivel \\
\hline 1 & Sistema & El proyecto responde a una ideación como sistema o como serie informativa & Afecta a los niveles inferior, medio y superior \\
\hline 2 & Datos & Codificación de datos, metadatos e información periodística & Nivel inferior \\
\hline 3 & Acceso & $\begin{array}{l}\text { Diseño de las capas que organizarán y presentarán las unidades informativas } \\
\text { y permitirán el acceso a los contenidos }\end{array}$ & Nivel medio \\
\hline 4 & Interactividad & $\begin{array}{l}\text { Diseño y organización del contenido y la estructura de menús que deben } \\
\text { facilitar la experiencia del usuario }\end{array}$ & Afecta a los niveles inferior, medio y superior \\
\hline 5 & Interfaz & $\begin{array}{l}\text { Definición de la interfaz en la que se concreta el acceso a los elementos de } \\
\text { interacción y navegación }\end{array}$ & Nivel superior \\
\hline
\end{tabular}

la información el usuario precisará de capas en las que esa información se organice semánticamente, así como en capas sintácticas, formando relatos narrativos o en capas en las que el contenido se ordene según caracterizaciones o morfologías. Son ejemplos de ello el diseño de una determinada visualización, la ordenación de contenidos y campos de datos en columnas y filas, la organización de índices o la concatenación de contenidos. Los autores deben crear y definir esas capas o utilizar herramientas prediseñadas.

\subsection{Interacción. Lectura personalizada}

La selección de las capas de información que van a formar parte de una pieza debe responder a un diseño previo de interacción en el que los autores hayan considerado lo que el usuario podrá explorar, leer o consultar. El diseño de la interacción de la pieza debe contemplar la potencialidad de los datos obtenidos, el rol que se quiere dar al usuario -lector, co-creador, participante, etc.-, así como la eficacia de las capas que se van a utilizar.

\subsection{Interfaz}

La interfaz se sitúa en el nivel superior de la pieza informativa, la del sistema. En ella se concreta y distribuyen las capas de información y los elementos de navegación e interacción que permitirán al usuario acceder a los contenidos, recorrer la pieza o participar en ella.

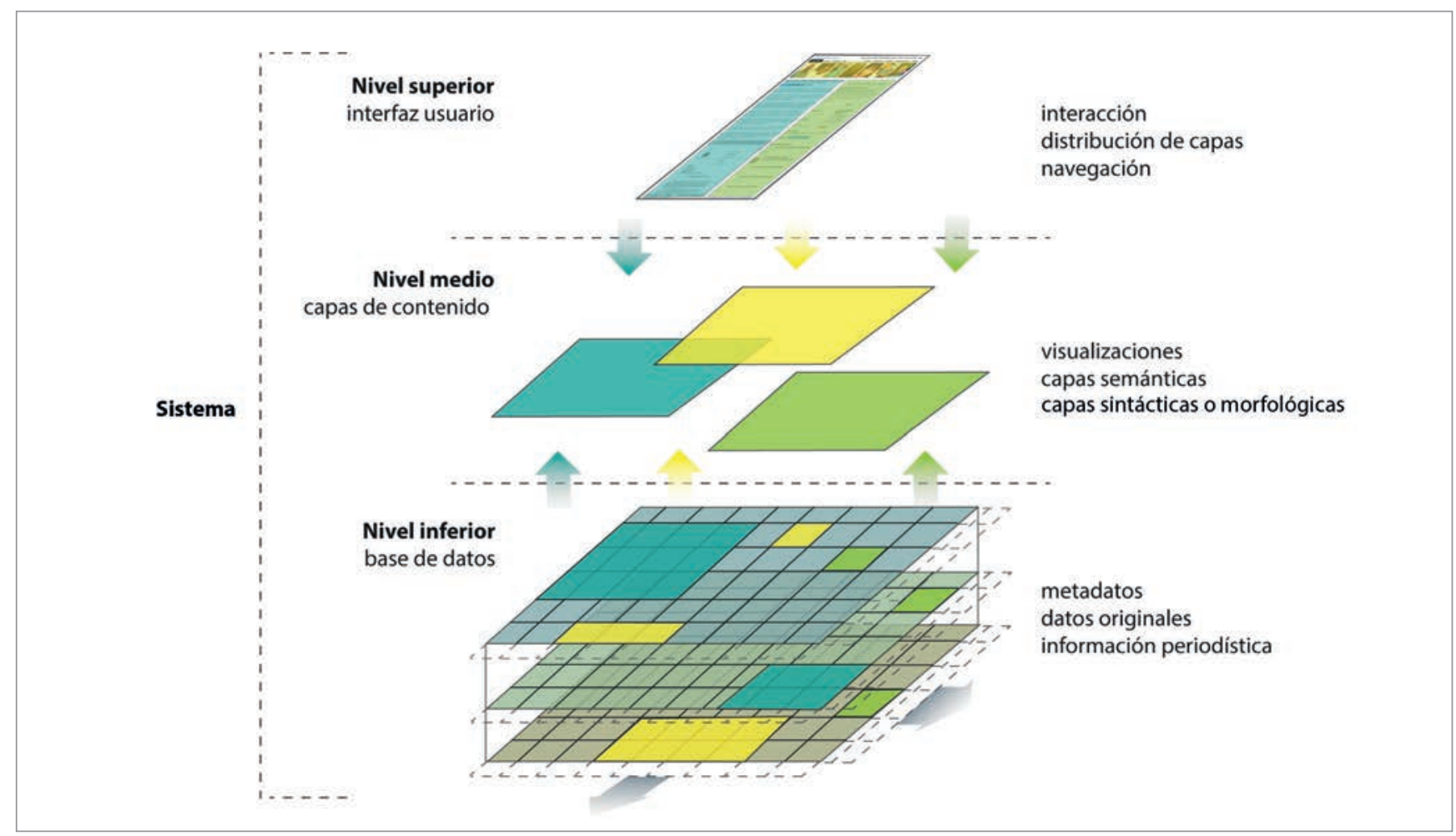

Figura 10. Representación del sistema de PE en tres niveles 
Datos codificados, capas de información e interfaz forman el conjunto de tres niveles que contiene todo sistema de periodismo estructurado (figura 10). Todos los proyectos presentados como ejemplos de buenas prácticas pueden analizarse desde esta perspectiva sistémica.

Todos ellos responden a una estructura de tres niveles en que el nivel central, el de las capas informativas, actúa de enlace entre la interfaz de navegación del usuario y la base de datos que contiene la información codificada. Es tarea de los autores definir cómo van a ser esas capas informativas acorde con la codificación de información que se deba realizar y con la interfaz y experiencia interactiva que se quiera dar al usuario. Todos ellos comparten también mecanismos de actualización de la información y retroalimentación que perduran un espacio de tiempo, el ciclo de vida informativo que se quiera dar a la pieza.

La mayoría de proyectos de PE están diseñados para prolongarse indefinidamente, manteniendo en todo momento su capacidad de informar sobre el presente

\section{Conclusiones}

La evolución del periodismo estructurado desde el mashup de Holovarty Chicagocrime.org de 2005 hasta Documenting hate, promovido por ProPublica y un consorcio de $80 \mathrm{em}$ presas, universidades y medios, de 2017 y aún en proceso de elaboración, permite trazar un recorrido por un conjunto importante de piezas informativas propias del escenario digital. Todas ellas se sitúan en la confluencia del periodismo de investigación, el diseño de interacción y el tratamiento digital de datos. En esta investigación hemos intentado poner de relieve la importancia de esos tres ámbitos en la definición del PE, a pesar de que algunos autores o proyectos asignen mayor o menor peso a cada una de sus partes.

Resulta interesante comprobar cómo el diseño de un sistema de codificación de información como Documenting hate o Structured stories no presuponen de por sí la creación de un sistema de información eficaz. Igualmente, la elección de un conjunto de capas de información, visualizaciones y herramientas interactivas pueden proporcionarnos un producto interactivo atractivo, pero si carece de un sistema de información que lo retroalimente y lo defina, no podemos hablar propiamente de PE.

El periodismo estructurado forma parte del sistema informacional. A pesar de que las previsiones más ambiciosas de Reg Chua quizá no se hayan podido llevar a cabo, hay buenos casos de éxito tanto dentro como fuera de los medios convencionales. En la mayoría de ejemplos se trata de proyectos de periodismo de investigación que requieren de un proceso largo de recopilación de datos, que se basan en una estrategia de acumulación para sumar valor informacional.

Su eficacia y éxito radica en la capacidad que ha mostrado para incorporarse paulatinamente en los sistemas de información de la mayoría de agentes dedicados a la información y su capacidad por convivir con otras formas de periodismo, a las que complementa y nutre. Como herramienta complementaria en The Washington Post o monitorizando el proceso electoral en Electionland en un consorcio de más de 250 organizaciones, empresas y medios, el PE supone una de las mayores transformaciones que el periodismo ha vivido desde que empezó el proceso de convergencia digital.

\section{Datos codificados, capas de información e interfaz forman el conjunto de tres ni- veles que contiene todo sistema de pe- riodismo estructurado}

Creemos que es muy importante profundizar en esta clase de modelos con capacidad potencial para volver a poner el periodismo profesional en el centro de la información social. Si existe un consenso claro entre los estudiosos, es precisamente en relación con esta necesidad. Si el periodismo no consigue recuperar o volver a afianzar su papel de cuarto poder, las sociedades democráticas pierden uno de sus principales pilares.

\section{Nota}

Este trabajo forma parte de las investigaciones sobre el ciclo de la vida informativa y los formatos y narrativas digitales interactivas, objetivos Ob1 y Ob2 del proyecto Creación y contenido interactivo en la comunicación de información audiovisual: audiencias, diseño, sistemas y formatos CSO201564955-C4-2-R (Mineco/Feder), Ministerio de Economía y Competitividad (España).

El PE supone una de las mayores transformaciones que el periodismo ha vivido desde que empezó el proceso de convergencia digital

\section{Referencias}

Adair, Bill (2015). "Structured stories NYC: An experiment in local news". Duke reporters' lab, 27 mayo.

https://reporterslab.org/structured-stories-nyc-an-experimentin-local-news

Amico, Chris (2013). "Reuters bets big on context, structure and dataviz to understand power in China". Nieman Lab, 24 April. http://www.niemanlab.org/2013/04/reuters-bets-big-oncontext-structure-and-dataviz-to-understand-power-in-china

Amico, Chris; Amico, Laura (2011). "About Homicide watch D.C.". Homicide watch D.C. http://homicidewatch.org/about

Amico, Laura (2015). "We remember: Four years of Homicide watch DC". Homicide watch D.C., 7 May.

http://homicidewatch.org

BBC News Lab (2015). "A manifesto for structured journalism". BBC News Lab, 7 July.

http://bbcnewslabs.co.uk/2015/07/07/a-manifesto-forstructured-journalism 
Bradshaw, Paul (2008). "Basic principles of online journalism: C is for community \& conversation (pt2: Conversation)". Online journalism blog, 18 Sept.

https://goo.gl/kozKzN

Caswell, David; Dörr, Konstantin (2017). "Automated journalism 2.0: Event-driven narratives". Journalism practice, 9 May.

http://www.zora.uzh.ch/id/eprint/137060/1/Automated_ Journalism_2.0_EventDriven_Narratives.pdf https://doi.org/10.1080/17512786.2017.1320773

Caswell, David; Russell, Frank; Adair, Bill (2015). “Editorial aspects of reporting into structured narratives". En: Procs of the 2015 Computation+ journalism symposium, New York, 2-3 October.

http://cj2015.brown.columbia.edu/papers/editorial.pdf

Chua, Reg (2010a). "Structured journalism". (Re)structuring journalism rethinking journalism and the business of journalism from the ground up, 12 August.

https://structureofnews.wordpress.com/2010/08/12/ structured-journalism

Chua, Reg (2010b). "What's it all about?". (Re)structuring journalism rethinking journalism and the business of journalism from the ground up, 10 August.

https://structureofnews.wordpress.com/2010/08/10/ whats-it-all-about

Cleveland, William S. (1993). Visualizing data. Hobart Press. ISBN: 0963488406

Cohn, David (2014). "At Circa, it's not about 'chunkifying' news but adding structure". Poynter, 7 Febr.

http://www.poynter.org/2014/circa-not-chunk-y-fyingnews-but-rather-adding-structure/237862

Craig, Heather (2015). Interactive data narrative: Designing for public engagement. Massachusetts Institute of Technology. http://cmsw.mit.edu/interactive-data-narrative-designingfor-public-engagement

Daniel, Anna; Flew, Terry (2010). "The Guardian reportage of the UK MP expenses scandal: A case study of computational journalism". En: Communications policy and research forum 2010, Sidney, 15-16 Nov.

https://eprints.qut.edu.au/38701/2/38701.pdf

Flew, Terry; Spurgeon, Christina; Daniel, Anna; Swift, Adam (2012). "The promise of computational journalism". Journalism practice, v. 6, n. 2, pp. 157-171.

https://goo.gl/wfaE4c

https://doi.org/10.1080/17512786.2011.616655

Flores-Vivar, Jesús-Miguel; Salinas-Aguilar, Cecilia (2012). "Entornos y perfiles profesionales para redacciones con cultura de red: mashups y data delivery editor (Datajournalism)". En: Comunicación y riesgo: III Congreso Internacional de la Asociación Española de Investigación en Comunicación, Tarragona, 18-20 enero.

http://www.aeic2012tarragona.org/comunicacions_cd/ ok/194.pdf

Freixa, Pere (2015). "Reportajes especiales en los cibermedios. Análisis de diez años de Premios Goya y Oscar Awards en Elpais.com (2005-2014)". El profesional de la información, v. 24, n. 3, pp. 291-300.

http://dx.doi.org/10.3145/epi.2015.may.09

Gillmor, Dan (2006). We the media: Grassroots journalism by the people for the people. O'Relly Media. ISBN: 9780 596102272

Gourarie, Chava (2015). "'Structured journalism' offers readers a different kind of story experience". Columbia journalism review, 30 July.

https://www.cjr.org/innovations/structured_journalism.php

Gray, Jonathan; Chambers, Lucy; Bounegru, Liliana. (2012). The data journalism handbook: How journalists can use data to improve the news. O'Reilly Media, Inc. ISBN: 9781 449330026

Grinapol, Corinne (2016). "ProPublica has 250 news organizations signed up for its Electionland project". Adweek, 19 Sept.

http://www.adweek.com/digital/propublica-has-250-newsorganizations-signed-up-for-its-electionland-project

Hamilton, James T.; Turner, Fred (2009). Accountability through algorithm: Developing the field of computational journalism. Technical report. Center for Advanced Study in the Behavioral Sciences.

https://goo.gl/i8pkLg

Hepworth, Shelley (2016). "300 newsrooms sign on to monitor voting problems". Columbia journalism review, 2 Nov. https://www.cjr.org/covering_the_election/electionland_ voting.php

Holovarty, Adrian (2006). "A fundamental way newspaper sites need to change". Adrian Holovarty blog, 6 Sept. http://www.holovaty.com/writing/fundamental-change

Holovarty, Adrian (2008). "In memory of Chicagocrime. org". Adrian Holovarty blog, 31 Jan.

http://www.holovaty.com/writing/chicagocrime.org-tribute

Kennedy, John (2013). "Reuters' China power map is totally awesome and somewhat informative". South China morning post, 8 March.

https://goo.gl/ZdcLBp

Larrondo, Ainara (2010). "The potential of web-only feature stories. A case study of Spanish media sites". Journalism studies, v. 12, n. 2, pp. 188-204.

https://goo.gl/7bNDUq

https://doi.org/10.1080/1461670X.2010.491300

Lewis, Seth C.; Westlund, Oscar (2015). "Big data and journalism: Epistemology, expertise, economics, and ethics". Digital journalism, v. 3, n. 3, pp. 447-466.

https://apps.cla.umn.edu/directory/items/publication/324155. pdf

https://doi.org/10.1080/21670811.2014.976418

Lichterman, Joseph (2016). "The New York Times is shutting down its lauded, younger-reader-seeking NYT now app". Nieman Lab, 18 August. https://goo.gl/ZpwS1V

Newton, Casey (2015). "Why Circa failed". The verge, 24 June. 
https://www.theverge.com/2015/6/24/8842009/whycirca-failed

OHern, Matthew S.; Rindfleisch, Aric (2010). "Customer co-creation". Review of marketing research, v. 6, pp. 84-106. ISBN: 9780857247285

http://www.emeraldinsight.com/doi/abs/10.1108/S15486435\%282009\%290000006008

Owen, Laura H. (2015). “'Learning to write again': A Duke team tests a new way of reporting on New York City government". Nieman Lab, 26 June.

https://goo.gl/pKu6y9

Pisani, Francis (2006). "Journalism and web 2.0". Nieman reports, $15 \mathrm{Dec}$.

http://niemanreports.org/articles/journalism-and-web-2-0

Popper, Daniel (2015). "Inside Billy Penn with CEO Jim Brady". American journalism review, 18 May.

http://ajr.org/2015/05/18/inside-billy-penn-with-ceo-jim-brady

ProPublica (2016). "Documenting hate. About". ProPublica, 8 Sept.

https://projects.propublica.org/graphics/hatecrimes

Qiu, Yue; Zhang, Wenxiong (2013). “Connecting China, visually. Reporters help tell Chinese political stories in an interactive way". Columbia journalism review, 15 April.

http://archives.cjr.org/data_points/connecting_china_ visually.php?page $=$ all

Ribas, Joan-Ignasi; Freixa, Pere (1997). “Disseny interactiu: una especialitat central en la producción de sistemes interactius multimèdia". Treballs de comunicació, v. 8, pp. 19-37. http://publicacions.iec.cat/repository/pdf/00000162/00000038. $p d f$

Sánchez-Bonvehí, Clàudia; Ribera, Mireia (2014). "Visualización de la información en la democratización de los datos: propuestas desde el periodismo y la narratividad". El profesional de la información, v. 23, n. 3, pp. 311-318. https://doi.org/10.3145/epi.2014.may.11

Soler-Adillon, Joan; Sora, Carles; Freixa, Pere; Ribas, Joan-Ignasi (2016). "Perfil del profesional de la comunicación interactiva: fundamentos, actualidad y perspectivas". El profesional de la información, v. 25, n. 2, pp. 196-208.

http://dx.doi.org/10.3145/epi.2016.mar.06

Spoerri, Anselm (2007). "Visual mashup of text and media search results". En: Information Visualization 2007. IV'07. $11^{\text {th }}$ Int l conf, pp. 216-221.

https://doi.org/10.1109/IV.2007.125

Stake, Robert E. (1995). The art of case study research. Sage Publications. ISBN: 9780803957671

Stearns, Josh (2015). "The best online storytelling and journalism of 2014". Medium, 5 Jan.

https://medium.com/@jcstearns/the-best-onlinestorytelling-and-journalism-of-2014-62a30e20575c

The New York Times (2017). "Why we need a project to document hate crimes". Editorial board: Opinion pages. The New York Times, 17 Jan.

https://www.nytimes.com/2017/01/17/opinion/why-weneed-a-project-to-document-hate-crimes.html

Trewinnard, Tom (2017). "Meedan joins documenting hate". Medium, 19 Jan.

https://medium.com/meedan-updates/meedan-joinsdocumenting-hate-d71364aa20d6

WashPosPR (2015). "The Washington Post tests new 'Knowledge map' feature”. The Washington Post, 16 July. https://goo.gl/tvqP2u

Wojcik, Laura (2015). "Structured journalism, the nest revolution in storytelling?". Medium, 4 October.

https://medium.com/interactive-journalism/structuredjournalism-the-next-revolution-in-storytelling-91557c48c121

Yin, Robert K. (2013). Case study research: Design and methods. $5^{\text {th }}$ edition, Sage. ISBN: 9781483322247



